\title{
Infrared space astrometry project JASMINE
}

\author{
N. Gouda ${ }^{1}$, Y. Kobayashi ${ }^{1}$, Y. Yamada ${ }^{2}$, T. Yano $^{1}$ \\ and JASMINE Working Group
}

\author{
${ }^{1}$ National Astronomical Observatory of JAPAN, Mitaka, Tokyo, 181-8588, JAPAN \\ email:naoteru.gouda@nao.ac.jp, yuki@merope.mtk.nao.ac.jp, yano.t@nao.ac.jp \\ ${ }^{2}$ Department of Physics, Kyoto University, Kyoto, 606-8502, JAPAN \\ email:yamada@scphys.kyoto-u.ac.jp
}

\begin{abstract}
A Japanese plan of an infrared (z-band:0.9 $\mu$ as or k-band:2.2 $\mu$ as) space astrometry (JASMINE - project) is introduced. JASMINE (Japan Astrometry Satellite Mission for INfrared Exploration) will measure distances and tangential motions of stars in the bulge of the Milky Way. It will measure parallaxes, positions with an accuracy of $10 \mu$ as and proper motions with an accuracy of $10 \mu$ as/year for stars brighter than $\mathrm{z}=14 \mathrm{mag}$ or $\mathrm{k}=11 \mathrm{mag}$. JASMINE will observe about ten million stars belonging to the bulge component of our Galaxy. With a completely new "map" of the Galactic bulge, it is expected that many new exciting scientific results will be obtained in various fields of astronomy. Presently, JASMINE is in a development phase, with a targeted launch date around 2016. Science targets, preliminary design of instruments, observing strategy, critical technical issues in JASMINE and also Nano-JASMINE project are described in this paper.
\end{abstract}

Keywords. infrared: general, space vehicles, astrometry, Galaxy: bulge

\section{Outline and scientific targets of JASMINE mission}

\subsection{JASMINE project}

JASMINE is an astrometric mission that observes in an infrared (z-band: central wavelength is $0.9 \mu \mathrm{m}$, or $\mathrm{k}$-band: central wavelength is $2.2 \mu \mathrm{m})$. The $\mathrm{k}$-band is a preferable candidate at the present time. It is designed to perform a survey towards the Galactic bulge, determining positions and parallaxes accurate to $\sim 10 \mu$ as for stars brighter than $\mathrm{z}=14 \mathrm{mag}$ or $\mathrm{k}=11 \mathrm{mag}$, and proper motion errors of $\sim 10 \mu \mathrm{as} / \mathrm{yr}$. JASMINE was originally proposed to survey the whole Galactic plane $\left(360^{\circ} \times 7^{\circ}\right)$ with both a primary mirror of $\sim 1.5 \mathrm{~m}$ and a beam combiner(Gouda et al.(2004)). Now, a new version of JASMINE is proposed that only performs a survey towards the Galactic bulge, with a single-beam telescope. Investigations of the Galactic bulge are very important to clarify the structure and formation history of the Galaxy, the evolution of types of galaxies (e.g. spiral galaxy versus elliptical galaxy), the activity of the Galactic center and so on.

JASMINE can detect about one million bulge stars with $\sigma_{\pi} / \pi \leqslant 0.1$, about 1000 times more than the number of stars measured by Hipparcos (about 400 stars) in the survey area of JASMINE (see Fig. 1). Here $\pi$ is a parallax of a star and $\sigma_{\pi}$ is the observational error of the parallax. This was derived using our Galactic model whose original version was made by Cohen and his collaborators(Wainscoat et al.(1992)).

JASMINE will adopt a "frame-link" method which is explained in section 3. This method can be used when the number of stars in each field-of-view(small-field) is so large that a frame of the whole survey region can be made by the combined small-fields with the required accuracy. The star density in the Galactic bulge is found to be sufficient to apply this method. 
There exist a number of major technical problems, such as telescope pointing stability, and thermal stability of the instruments. Investigations proceed in collaboration with JAXA(Japanese Aerospace Exploration Agency). A Lissajous orbit around the Sun-Earth Lagrange point L2 is a preferred option because this region provides a very stable thermal environment, minimization of eclipses, and so on. Also, other candidate orbits such as HCPO (High altitude Circular Polar Orbit) are studied. We hope that JASMINE will be launched around 2016. The mission lifetime will be 6 years.

\subsection{Science targets}

JASMINE will provide distances and transverse motions of stars in the Galactic bulge. Other space astrometry missions will accurately provide the structure of halo and disk of the Galaxy. Thus JASMINE will be complementary to Gaia, SIM and OBSS(refer to each URL in the reference list).

Bulges in spiral galaxies, similar in many ways to elliptical galaxies, are the key to study galaxy formation and the evolution of galaxy types. However, the size, shape and kinematical properties of the bulge in our Galaxy are pending problems. It is therefore important to investigate the 3 -dimensional positions and motions of stars in the Galactic bulge.

Some observations(e.g. Eckart \& Genzel(1996)) suggest that a super massive black hole exists in the center of the Galaxy; its growth seems strongly related to the growth of the bulge (e.g. Gebhardt et al.(2000)). Thus it is very interesting and important to clarify the growth of the bulge in the Galactic bulge. We believe these problems can be addressed, using the dynamical and kinematical structures obtained by JASMINE.

Furthermore the astrometric parameters of the many stars in the bulge, obtained by JASMINE, will have a large impact on the formations and evolutions of stars, and dust information in the Galactic bulge.

\section{Preliminary instrument design}

A candidate for the optics of the JASMINE telescope is a modified Korsch system with three mirrors and four folding flats to fit the focal length into the available volume. The telescope has a circular primary mirror with $\sim 75 \mathrm{~cm}$ diameter and $22.5 \mathrm{~m}$ (for z-band) or $12.3 \mathrm{~m}$ (for k-band) focal length.

The telescope provides a flat image plane that contains an array of large format detectors with a field of view of $0.7^{\circ} \times 0.7^{\circ}$. A total of $812 \mathrm{k} \times 2 \mathrm{k} \operatorname{CCDs}$ (for z-band) or 16 $2 \mathrm{k} \times 2 \mathrm{k} \mathrm{HgCdTe}$ (for $\mathrm{k}$-band) are read out.

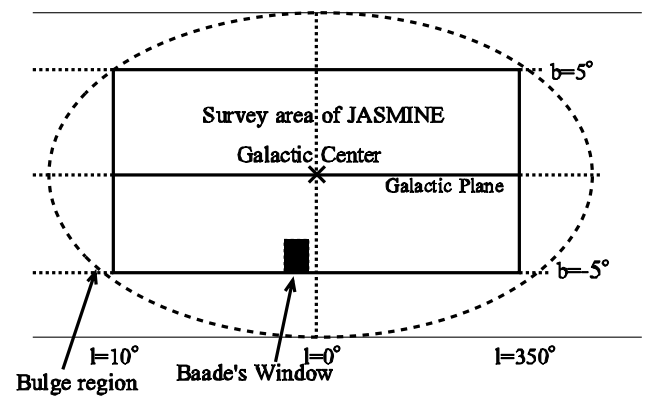

Figure 1. Survey area of JASMINE. 


\section{Observing strategy}

\subsection{Procedures of observation}

JASMINE has a single telescope with a $0.7^{\circ} \times 0.7^{\circ}$ field of view. One field of view will be observed during a few seconds. One set of stellar images observed in a few seconds is called a "small-frame". In such a small-frame the relative positions (centroids) of stars will be calculated using an algorithm that determines the weighted mean of the positions of the photons registered by $5 \times 5$ pixels.

The telescope moves toward an adjacent field of view (small-frame) overlapping the previous small-frame (the overlap area is about a half of the frame size). In about 10 hours the telescope takes stellar images over the whole survey region $\left(20^{\circ} \times 10^{\circ}\right)$, covering it by about 2000 small-frames. The small-frames are linked together by the many stars in the overlap regions. The whole survey region, linked together by small-frames is termed "a large-frame". The random error caused by linking small-frames is proportional to the number of small-frames in one-direction and inversely proportional to the square root of the number of common stars in the overlap region of each two adjacent small-frames. It is numerically found that the number density of measurable stars by JASMINE is large enough to attain the targeted accuracy of the link.

The above procedure is repeated during the whole mission life of about 6 years and finally about 10000 large-frames will be observed. Each large-frame covers the common survey area of $20^{\circ} \times 10^{\circ}$. Combining these large-frames allows to determine the 5 astrometric parameters with the targeted accuracy.

It should be noted that about a quarter of a year the direction towards the Sun from the spacecraft overlaps with that of the Galactic bulge. Observing the Galactic bulge is thus restricted to about $75 \%$ of the total mission life.

\subsection{Systematic errors and technical issues}

In this sub-section, systematic errors that may affect JASMINE are briefly reviewed. Large systematic errors can occur in making a large-frame because small-frames may have different sizes and distortions due to variations of the telescope geometry according to temperature variations around the telescope. Hence very small temperature variations around the telescope and also minimal changes in telescope's geometry are mandated to reduce the systematic error. The use of QSOs measurable by JASMINE in the survey region will relax the requirement to the satellite system and the telescope. However variations of the telescope geometry smaller than about $30 \mathrm{pm}$ within around 10 hours are required. This requirement will be reached by using ultra-low expansion optics and thermal structure of the satellite that ensure high stability of temperature around the telescope. These technical issues are now under investigation. Furthermore it is necessary to monitor small variations of the telescope geometry with high accuracies in order to verify the required small variations. Such monitoring systems are developed using laser interferometer technology.

Long term-contributors to JASMINE's error budget include changes in the scale of large-frames. This effect can be calibrated by QSOs.

There exist other systematic errors caused by radiation damage to the detectors, aberration, multiple stars, gravitational lensing effects, but these effects can be calibrated.

One QSO measurable by JASMINE has been already found. We have 14 QSO candidates measurable by JASMINE. Their true nature will be confirmed by planned spectroscopic observations. 


\section{Nano-JASMINE project}

A Nano-JASMINE project is planned to demonstrate space astrometry in Japan and to examine some technical issues for JASMINE (Kobayashi et al.(2006);Suganuma et al.(2006)).Nano-JASMINE uses a nano-satellite whose size and weight are about 40 $\mathrm{cm}^{3}$ and $14 \mathrm{~kg}$, respectively. The size of the telescope is reduced to a $5 \mathrm{~cm}$ aperture and a focal length of about $1.7 \mathrm{~m}$. One CCD with $1 \mathrm{k} \times 1 \mathrm{k}$ pixels for $\mathrm{z}$-band will be put in the focal plane. A candidate orbit for Nano-JASMINE is a sun-synchronous orbit. We will examine damage due to radiation on the $\mathrm{CCD}$, on-board processing, thermal variations of the optical system and so on. The cost of Nano-JASMINE is low and it is expected to be launched around 2009. The development of the spacecraft is ongoing, in collaboration with Prof. Nakasuka and his group at the University of Tokyo.

\section{Management and schedule}

The establishment of a JASMINE working group at the JAXA was approved in 2003 by a science committee of ISAS (Institute of Space and Astronautical Science) of JAXA. JASMINE project office has been established at the NAOJ (National Astronomical Observatory of Japan) in 2004. We aim at a proposal for the JASMINE mission to JAXA, to get the launch approval and the required budget from the Japanese government about 4 years later. We hope that JASMINE will be launched around 2016. It may take long time to get the accurate astrometric parameters in the Galactic bulge. We would like to ask you for your continuous encouragement and cooperation.

\section{References}

Eckart, A. \& Genzel, R. 1996, Nature 383, 415.

Gebhardt, K., Karl, B., Ralf, B., Gary, D., Alan, F., Faber, S. M., Filippenko, A. V., Green, R., Grillmair, C., Carl, H., Luis, C., Kormendy, J., Lauer, T. R., Magorrian, J., Pinkney, J., Richstone, D., \& Tremaine, S. 2000, 'ApJ, 539, L13, 2000.

Gouda, N., Yano, T. , Kobayashi, Y., Yamada, Y., Tsujimoto, T., Nakajima, T., Suganuma, M., Matsuhara, H., Ueda, S. \& JASMINE Working Group. 2004, in:D.W.Kurtz(eds.), Transits of Venus: New Views of the Solar System and Galaxy, Proc.IAU Symposium No.196(Cambridge University Press), p. 455.

Kobayashi, Y., Gouda, N., Tsujimoto, T., Yano, T., Suganuma, M., Yamauchi, M., Takato, N., Miyazaki, S., Yamada, Y., Sako, N., \& Nakasuka, S. 2006, in Proceedings of the SPIE, 6265 J. Mather etc., ed., 626544-1-626544-10.

Suganuma, M., Kobayashi, Y., Gouda, N., Yano, T., Yamada, Y., Takato, N., \& Yamauchi, M. 2006, in Proceedings of the SPIE, 6265 J. Mather etc., ed., 626545-1-626545-12.

Wainscoat, R. J., Cohen, M., Volk, K., Walker, H. J. \& Schwartz, D. E. 1992, ApJS, 83, 111-146.

GAIA:http://www.rssd.esa.int/index.php?project=GAIA

SIM:http://planetquest.jpl.nasa.gov/SIM/

OBSS:http://ad.usno.navy.mil/OBSS/

JASMINE:http://www.jasmine-galaxy.org/index.html 